\title{
A CALIBRATED, NON-INVASIVE METHOD FOR MEASURING THE INTERNAL INTERFACE HEIGHT FIELD AT HIGH RESOLUTION IN THE ROTATING, TWO-LAYER ANNULUS
}

\author{
P.D. WILLIAMS ${ }^{\mathrm{a}, *}$, P.L. READ ${ }^{\mathrm{a}}$ and T.W.N. HAINE ${ }^{\mathrm{b}}$ \\ ${ }^{a}$ Atmospheric, Oceanic and Planetary Physics, Oxford University, UK; \\ ${ }^{\mathrm{b}}$ Department of Earth and Planetary Sciences, Johns Hopkins University, Baltimore, USA
}

(Received 29 January 2004; In final form 15 July 2004)

\begin{abstract}
We describe a remote sensing method for measuring the internal interface height field in a rotating, two-layer annulus laboratory experiment. The method is non-invasive, avoiding the possibility of an interaction between the flow and the measurement device. The height fields retrieved are accurate and highly resolved in both space and time. The technique is based on a flow visualization method developed by previous workers, and relies upon the optical rotation properties of the working liquids. The previous methods returned only qualitative interface maps, however. In the present study, a technique is developed for deriving quantitative maps by calibrating height against the colour fields registered by a camera which views the flow from above. We use a layer-wise torque balance analysis to determine the equilibrium interface height field analytically, in order to derive the calibration curves. With the current system, viewing an annulus of outer radius $125 \mathrm{~mm}$ and depth $250 \mathrm{~mm}$ from a distance of $2 \mathrm{~m}$, the inferred height fields have horizontal, vertical and temporal resolutions of up to $0.2 \mathrm{~mm}, 1 \mathrm{~mm}$ and $0.04 \mathrm{~s}$, respectively.
\end{abstract}

Keywords: Two-layer annulus; Interface height; Optical activity; Torque balance; Calibration

\section{INTRODUCTION}

Since the nineteenth century, laboratory experiments have been performed on rotating fluids in order to improve our understanding of geophysical fluid dynamics. In the laboratory, as for the atmosphere and ocean, techniques have been developed for making detailed measurements of the flow, which are essential for a quantitative comparison with theory. For example, laboratory techniques for measuring fluid velocities have become quite advanced. By adding small, neutrally buoyant beads to the working liquid, sophisticated particle tracking techniques can be used to derive velocity fields,

\footnotetext{
*Corresponding author. Present address: Centre for Global Atmospheric Modelling, Department of Meteorology, University of Reading, UK. E-mail: williams@atm.ox.ac.uk
} 
$\boldsymbol{u}(x, y, z, t)$, by measuring the displacements of individual beads over small time intervals (e.g., Fincham and Spedding, 1997). Ultrasonic doppler velocimetry techniques can also give reliable velocity measurements (e.g., Takeda, 1995). Methods for making accurate laboratory measurements of the height field of a liquid free surface, $z=h(x, y, t)$, have remained relatively elusive, however.

In a study of internal waves in a rotating liquid consisting of two stratified, immiscible layers, Hart (1972) used a vertical conducting wire stretched through the interface, acting as an in situ capacitative level sensor. One liquid was an electrical insulator and the other a slight conductor. The voltage drop across the wire could be calibrated against internal interface height but, with just a single probe, heights could be inferred at a single horizontal point only. Whilst this gives useful information about the temporal structure of the flow, the spatial structure is not retrieved. Furthermore, it is possible that the probe functions not simply as a passive measurement device, but as one which actively disturbs the flow.

Remote sensing methods, in which electromagnetic radiation emitted by or transmitted through the fluid is viewed non-invasively from afar, are generally preferred to in situ methods, to avoid interactions between the flow and the measurement device. Such interactions may have the potential to significantly disturb the flow. For example, Read (1992) inserted thermocouple probes into a rotating, thermal annulus, in order to make in situ measurements of the temperature field. Fast oscillations were recorded by the thermocouples, which appeared to be inertia-gravity waves and which contaminated the signal of interest. It is possible that the oscillations were generated by the presence of the probe.

The simplest remote sensing methods have problems which are difficult to overcome. With the global, non-invasive technique of shadowgraphy (Goldstein, 1983), in which parallel rays of light are passed vertically through a fluid interface and focused onto a screen by refraction at the disturbances, the problem of reconstructing the height field from the shadowgraph can be very difficult when the disturbances are so steep that the refraction is nonlinear. The Schlieren method (Goldstein, 1983) is impractical for similar reasons.

A sophisticated remote sensing flow visualization technique was developed by Hart and Kittelman (1986), to investigate the flow in a rotating cylinder containing two superposed, immiscible liquids. In that experiment, the lower liquid is chosen to be optically active, so that plane-polarized white light propagating vertically through the lower fluid has its plane of polarization rotated by an angle which depends upon both the wavelength and the lower layer depth. After leaving the fluids, the angularly-dispersed white light passes through a sheet of polaroid before being received by a colour camera. For a given lower layer depth, only light of a certain wavelength has its polarization axis rotated into exact alignment with the polaroid. Light of other wavelengths is either partially or fully extinguished by the polaroid, giving a correlation between interface height and colour registered by the camera. The quantitative calibration, to determine the functional relationship between height and colour, was not attempted by Hart and Kittelman. This meant that, though wavelengths and propagation speeds could easily be determined using this method, wave amplitudes could not, since information about the magnitude of interface height perturbations remained qualitative. The technique of using an optically active fluid to visualize a flow has also been employed by Ruddick (1991), in his laboratory model of double-diffusive interleaving at a thermohaline front. 
The purpose of the present article is to advance Hart and Kittelman's flow visualization technique, by deriving the height/colour calibration curve. This permits quantitative maps of interface height to be reconstructed, allowing wave amplitudes to be derived for the first time using this method. In Section 2, we describe the present rotating laboratory experiment, and discuss the calibration problem in more detail. In Section 3, we derive the nonlinear torque balance equations required for our calibration method, and show how solutions can be obtained iteratively. We derive the calibration curves in Section 4, and then give an example of an application of the calibration scheme by reconstructing the interface height from a colour laboratory image. Finally, we summarize the method and results, and discuss the limitations of our approach, in Section 5.

\section{THE ROTATING, TWO-LAYER ANNULUS}

The present apparatus is shown in Fig. 1. It consists of a cylindrical stainless steel tank of inner radius $125.0 \mathrm{~mm}$ and depth $250.0 \mathrm{~mm}$, which has a fixed glass base and a removable glass lid. A solid steel cylinder, of radius $62.5 \mathrm{~mm}$ and depth $250.0 \mathrm{~mm}$, is glued coaxially to the base of the tank to form an annulus of gap width $62.5 \mathrm{~mm}$. The annular region is filled to the brim with equal volumes of two immiscible liquids, to give a welldefined interface and equal resting layer depths of $125 \mathrm{~mm}$.

The tank is mounted centrally on a circular turntable which can be made to rotate under computer control with angular velocity $\Omega$. The annulus lid, which is in contact with the upper liquid, can also be made to rotate under computer control with angular velocity $\Delta \Omega$ relative to the tank. This is possible because the lid is connected to the tank via a ball race, allowing low-friction relative motion powered by a servo motor and drive wheel. The rotating lid forces a velocity shear across the fluid interface, providing an energy source from which baroclinic and/or Kelvin-Helmholtz instabilities can grow.

There is a central circular hole in the turntable, of radius equal to the tank radius, so that white light from a bright $500 \mathrm{~W}$ tungsten-halogen source lamp on the laboratory floor may pass vertically into, through and out of the annular gap. The light is received by a colour charge-coupled device (CCD) video camera, which is on the rotation axis and co-rotates with the turntable around $2 \mathrm{~m}$ above it. Communications between the laboratory frame and the rotating turntable frame (i.e. the camera power and output signal, and the servo motor power) are achieved through a commutator slip-ring, hidden from camera view by the inner cylinder. The S-VHS signal output by the CCD camera is recorded at 25 frames per second onto video tapes. The signal from the tapes can subsequently be input to a computer with a frame grabber, to produce colour 24-bit digitized images measuring 768 pixels by 576 pixels.

The upper layer is water, of density $997 \mathrm{~kg} \mathrm{~m}^{-3}$, and the lower layer is a blend of d-limonene and 1,1,2-trichlorotrifluoroethane (CFC-113), of densities $840 \mathrm{~kg} \mathrm{~m}^{-3}$ and $1570 \mathrm{~kg} \mathrm{~m}^{-3}$, respectively. The two lower layer constituents are mixed in such proportions that the two-layer liquid is slightly positively stratified, i.e. the upper layer is slightly less dense than the lower layer. Pure limonene is less dense than water, which is why it must be mixed with CFC-113 if it is to be the lower layer. This configuration is necessary to prevent harmful limonene vapours from evaporating into the laboratory air. The limonene/CFC mixture is hydrophobic, so that the two layers are mutually 


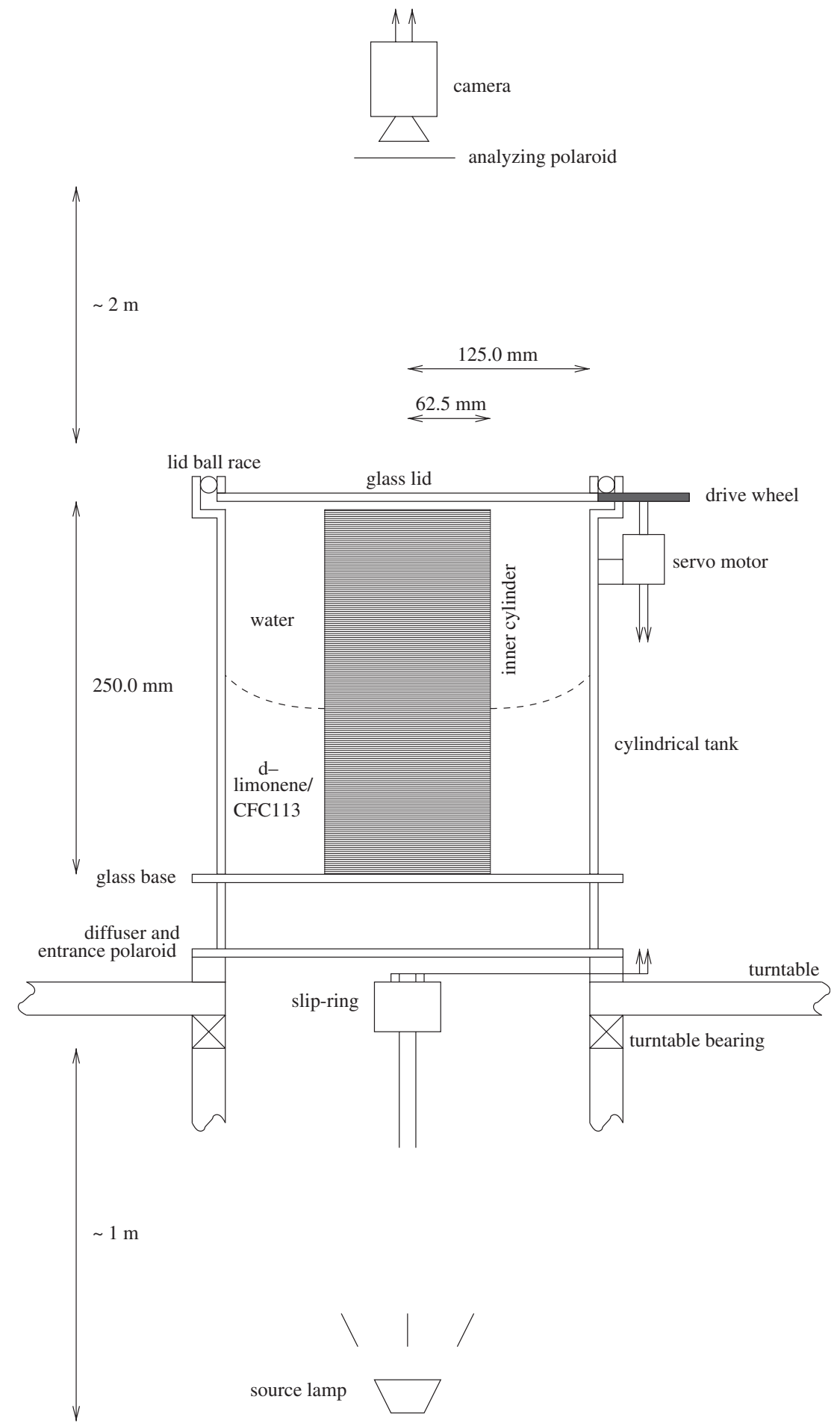

FIGURE 1 Schematic cross-section through the two-layer annulus apparatus, showing the principal mechanical components (not to scale). 
TABLE I Selected physical properties of the working liquids at the ambient laboratory temperature $\left(20^{\circ} \mathrm{C}\right)$.

\begin{tabular}{lcc}
\hline & $\begin{array}{c}\text { Water } \\
\text { Layer 1 } \\
\text { (Upper })\end{array}$ & $\begin{array}{c}\text { Limonene } / \text { CFC-113 } \\
\text { Layer 2 } \\
(\text { Lower })\end{array}$ \\
\hline Density, $\rho\left(\mathrm{kg} \mathrm{m}^{-3}\right)$ & $997 \pm 1$ & $1003 \pm 1$ \\
Mutual interfacial tension, $S\left(10^{-2} \mathrm{~N} \mathrm{~m}^{-1}\right)$ & $2.85 \pm 0.1$ & $2.85 \pm 0.1$ \\
Kinematic viscosity, $\left(10^{-6} \mathrm{~m}^{2} \mathrm{~s}^{-1}\right)$ & $1.27 \pm 0.02$ & $1.08 \pm 0.02$ \\
Optical activity, $\phi$, at $0.59 \mu \mathrm{m}^{\circ}\left(\mathrm{m}^{-1}\right)$ & 0 & $770 \pm 10$ \\
Refractive index, $n$, at $0.59 \mu \mathrm{m}$ & $1.3328 \pm 0.0001$ & $1.4466 \pm 0.0001$ \\
\hline
\end{tabular}

immiscible and give a well-defined interface. It is transparent and colourless, to allow the passage of light without significant absorption, and it has an optical activity which is non-zero and which varies strongly with wavelength for visible light (due to the limonene). Apart from the substitution of methylene bromide with CFC-113, and the change from cylindrical to annular geometry, the apparatus is similar to that used by Hart and Kittelman (1986). Selected fluid properties are shown in Table I. The slightly different refractive indices of the two layers means that light rays could deviate from the vertical when the interface is not horizontal. However, the effects of refraction at the interface have been evaluated and shown to be small (Williams, 2003) and so are neglected here.

As shown in Fig. 1, quasi-white light emitted by the source lamp travels vertically upwards through the apparatus and first passes through a diffuser. This is a translucent plastic sheet which diffuses the incoming light such that it illuminates the base of the tank uniformly. Without the diffuser, the video images would contain contrasting bright and dim regions, which would make interpretation and analysis more difficult.

The diffuse light passes through an entrance polaroid, fixed to the upper side of the diffuser. This is a thin sheet of linearly-polarizing filter. The direction of its polarization vector determines a vertical plane of polarization for the emerging light. Importantly, the entrance polaroid is fixed to the rotating turntable. This means that the vertical polarization plane of the light entering the fluids will rotate in the laboratory frame, but is fixed in the camera frame.

Next, the light enters the tank via its glass base, and travels through the optically active lower layer liquid, whose effect is to rotate the plane of polarization of the light. The rotation angle per unit depth for pure limonene has been determined experimentally by Hart and Kittelman (1986), for a range of wavelengths spanning the visible part of the spectrum. We derive the rotation angle for the present limonene/CFC-113 mixture, from Hart and Kittelman's pure limonene data, by assuming that it is reduced by a fraction equal to the volume-fraction of CFC-113 in the composite mix (i.e. $22 \%$ ). This assumption is easily verified theoretically by taking the total rotation angle to be the same whether the constituent liquids are well-mixed or are separated into distinct layers. The resulting optical activity curve is shown in Fig. 2.

Next, the light travels through the optically-inactive upper layer and leaves the tank via the glass lid, during which its plane of polarization is unchanged. The light then passes through an analyzing polaroid, which is a second thin sheet of linearly-polarizing filter fixed to the camera lens. This polaroid only allows the transmission of a certain fraction of the incident light intensity. Assuming perfect polaroids, this fraction 


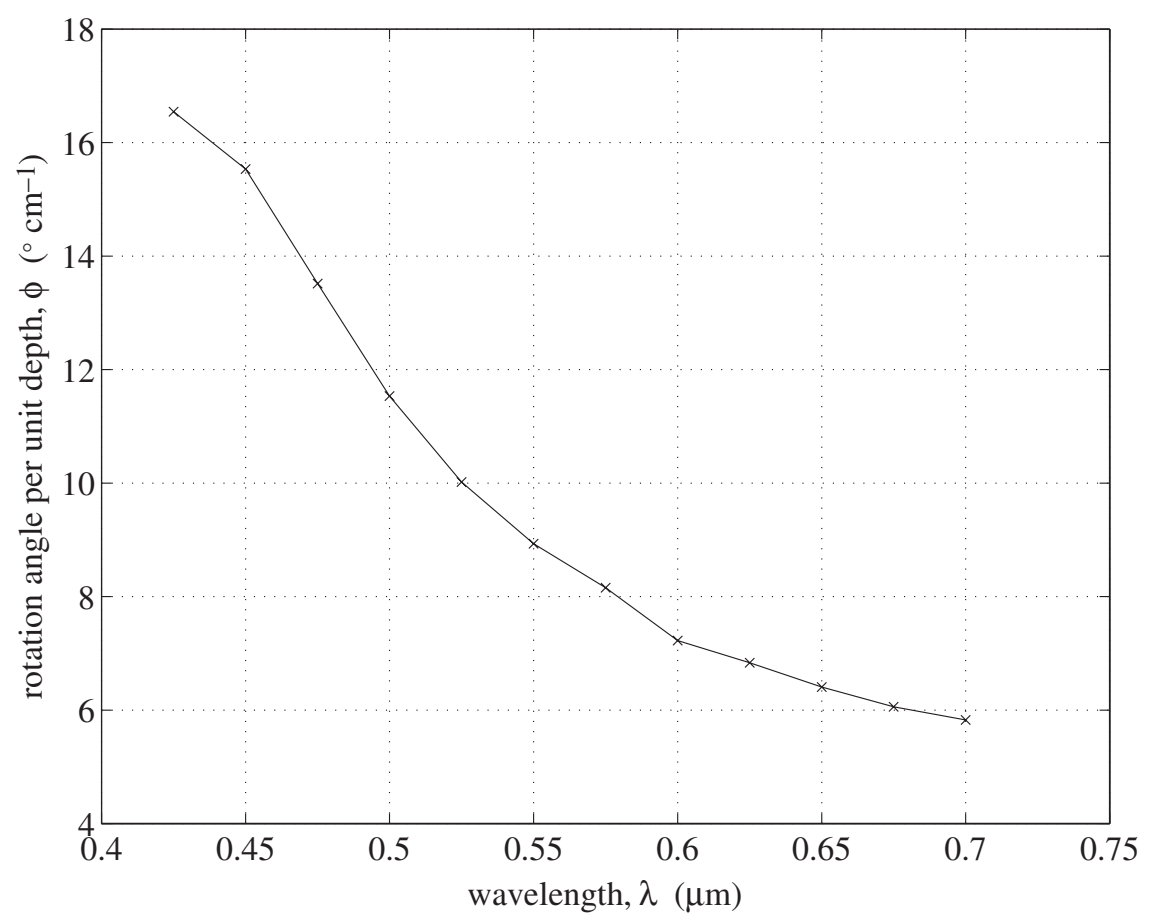

FIGURE 2 Optical rotary dispersion curve for the lower layer liquid of the present laboratory experiments, i.e. a blend of limonene and CFC-113, derived from the corresponding curve for pure limonene obtained by Hart and Kittelman (1986).

varies from 1 if the analyzing polaroid axis and incident light polarization axis are parallel (or anti-parallel), to 0 if they are perpendicular. For a given lower layer depth, therefore, only light of certain wavelengths will be rotated into close alignment with the analyzing polaroid and be transmitted to the camera. Other wavelengths will be extinguished by the polaroid. This is the origin of the relationship between lower layer depth, and colour recorded in the video images.

A typical scene captured by the camera is shown in Fig. 3. The rotation parameters are $\Omega=0.46 \mathrm{rad} \mathrm{s}^{-1}$ and $\Delta \Omega=3.70 \mathrm{rad} \mathrm{s}^{-1}$, at which there is a rotationally-modified Kelvin-Helmholtz instability but no baroclinic instability. The equilibrated flow is a Kelvin-Helmholtz mode of azimuthal wavenumber 9, which drifts around the annulus in the turntable frame at a speed of around half of the lid speed.

For a lower layer depth of $15 \mathrm{~cm}$, we deduce from Fig. 2 that the red light component $(\lambda \sim 0.70 \mu \mathrm{m})$ will be rotated through an angle of about $90^{\circ}$, the green light $(\lambda \sim 0.55 \mu \mathrm{m})$ through $135^{\circ}$ and the blue light $(\lambda \sim 0.44 \mu \mathrm{m})$ through $240^{\circ}$. These angles are shown in Fig. 4, where the angle between the axes of the crossed entrance and analyzing polaroids is taken to be $50^{\circ}$. In this case, most of the blue light will be transmitted through the analyzing polaroid, plus some of the red light but hardly any of the green light, and we would therefore see a predominantly blue colour for this height.

By rotating the analyzing polaroid attached to the camera lens, the angle between the axes of the crossed entrance and analyzing polaroids can be adjusted, which varies the colour observed for a given interface height. For some angles, the relationship between 


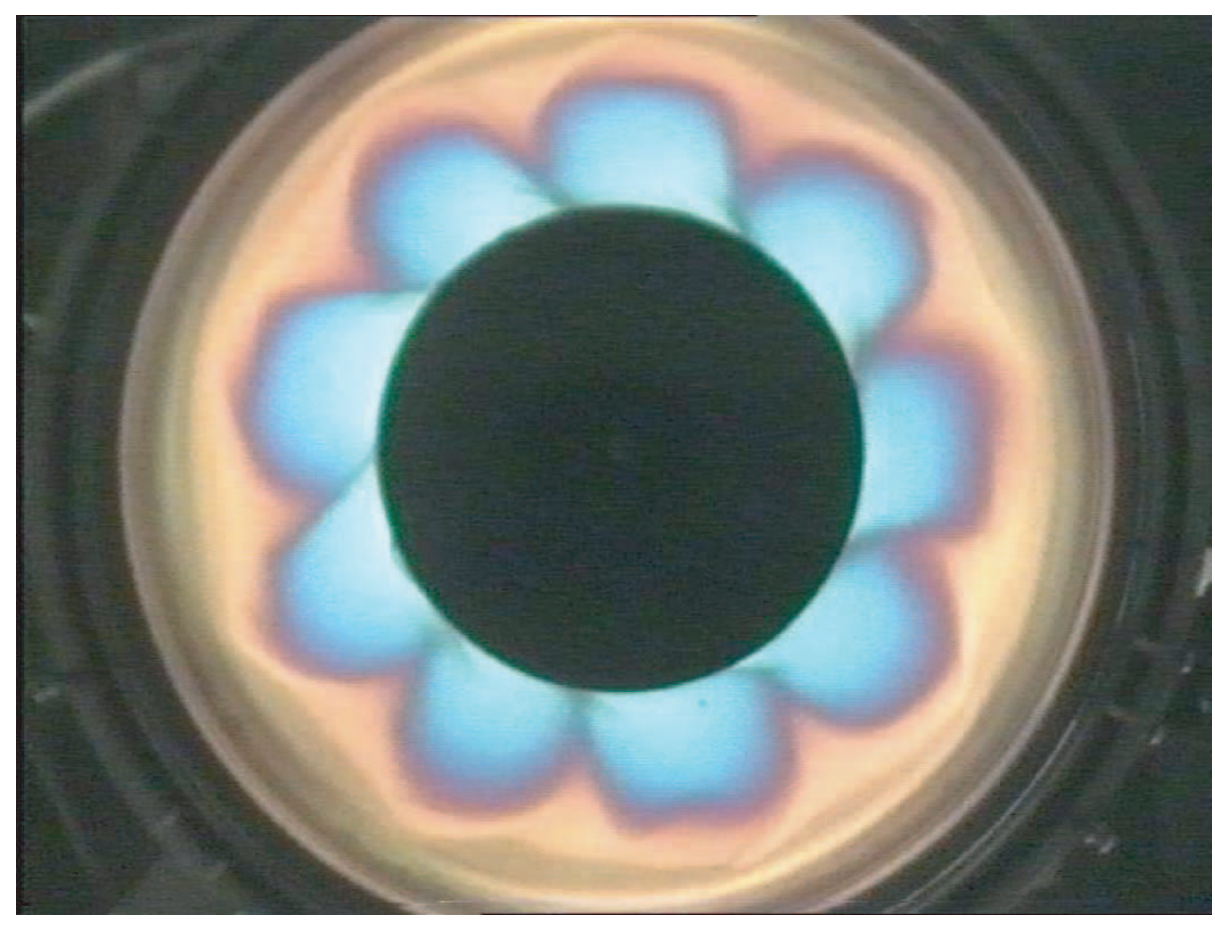

FIGURE 3 Typical laboratory image, captured by the video camera and digitized using a high-resolution frame grabber. Blue regions correspond to relatively large values of interface height, and yellow regions to relatively low values. The turntable and lid rotation speeds, $\Omega$ and $\Delta \Omega$, respectively, are both positive. Since the video camera which recorded this image is fixed in the turntable frame, the turntable appears to be stationary and the lid (and disturbances) rotate in the anti-clockwise sense.

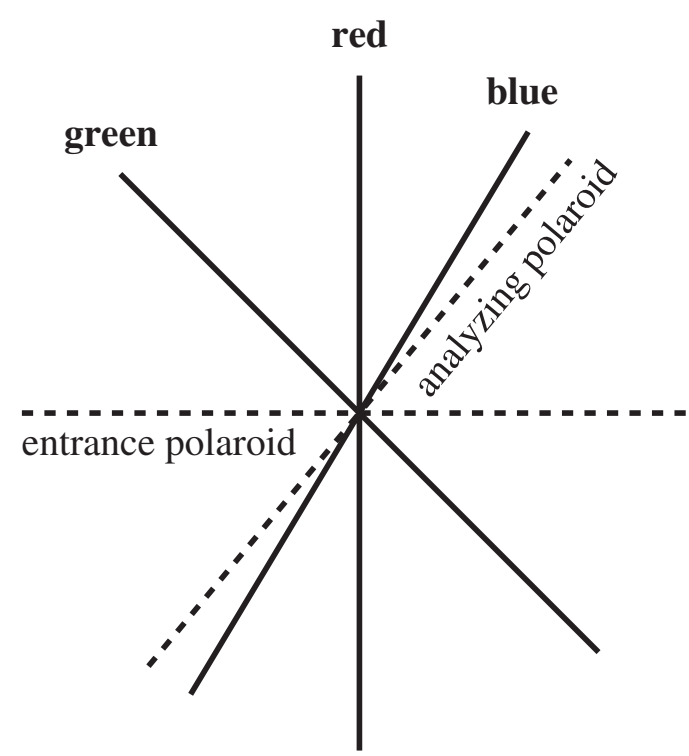

FIGURE 4 The polarization axes of red, green and blue light, after travelling through $15 \mathrm{~cm}$ of the limonene/CFC-113 mixture, as viewed from above by the video camera. The entrance and analyzing polaroids are shown as dashed lines. 
height and colour is more sensitive than for others, i.e. smaller changes in height produce larger changes in colour. In this study, the differential angle is adjusted - by trial and error - to be such that the relationship is at its most sensitive. This optimizes the vertical resolution, so that even small changes in interface height produce a significant signal in the colour field. The angle used is difficult to accurately obtain, because the two polaroids are over $2 \mathrm{~m}$ apart, and so no attempt was made to measure it.

It is our intention to calibrate the experiment by inferring the mathematical relationship between colour and height. It is not possible to derive the calibration curve experimentally, by increasing the depth of the lower layer in discrete steps and recording the colour at each one, since this method would expose the user to excessive quantities of the harmful limonene vapours.

The calibration requires an independent method for obtaining the interface height field in just one special case, when the experiment is in operation. The method must be independent, in the sense that it does not rely on the colour information in the images, since that is what we wish to calibrate. Fortunately, it is possible to derive an analytical expression for the equilibrium interface height in the special case of no baroclinic or Kelvin-Helmholtz instability, using layer torque balance equations. In this case, zonal wave modes are completely absent. The interface height is axisymmetric, but can still vary strongly with radius.

We plan to take interface height as a function of radius from the analytical expression, and colour as a function of radius from a laboratory experiment, and to determine the relationship between interface height and colour from the two, by eliminating the radius. We derive the required analytical expression in Section 3.

\section{TORQUE BALANCE ANALYSIS}

In this section, we seek an analytical expression for the stable equilibrium interface height shape, which we will use in Section 4 for the derivation of calibration curves. The interface shape obtained from the steady-state solution of the two-layer quasigeostrophic equations is not accurate enough for our purposes, as we will shortly show. We therefore choose an alternative and more accurate approach, based on setting to zero the net torque acting on each layer due to the thin, viscous boundary layers which surround it on all sides.

\subsection{Equilibrium interface height field}

Variable definitions for the torque balance calculation are shown in Fig. 5. In the turntable frame, which rotates with angular velocity $\Omega$ in the laboratory frame, the base $(z=0)$ and sidewalls $(r=a$ and $r=b=2 a)$ are stationary, and the lid $(z=2 H)$ rotates with angular velocity $\Delta \Omega$. The equilibrium flow in the fluid interiors is assumed to be completely azimuthal, i.e. to have neither a vertical nor a radial component. In reality, there is a small non-azimuthal secondary circulation throughout both fluid interiors, which controls the azimuthal flow (Proudman, 1956; Stewartson, 1966). The secondary circulation involves flow into and out of the Ekman layers, and vertical stretching and compression of fluid columns. The relative magnitude of this circulation is $\mathrm{O}\left(E^{1 / 2}\right)$, where $E=v /\left[\Omega(b-a)^{2}\right]$ is the Ekman number (Greenspan, 1968). Since we typically have $E \sim 10^{-4}$ in the present context, the departures from purely 


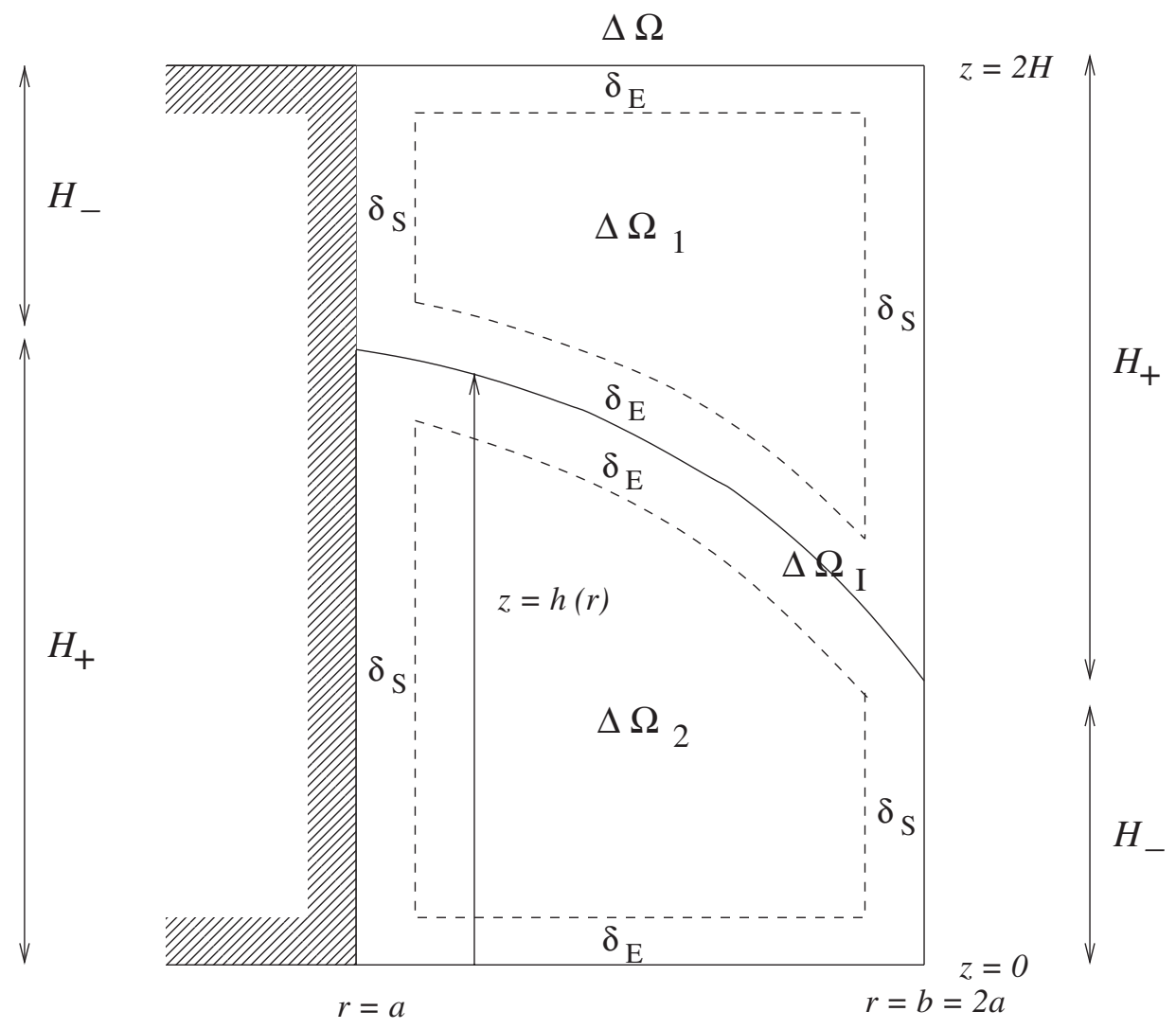

FIGURE 5 Schematic cross-section of the annulus, giving variable definitions for the torque balance calculation. The boundary layer widths, $\delta_{\mathrm{E}}$ and $\delta_{\mathrm{S}}$, are shown exaggerated, and the angular velocities are as observed in the turntable frame. See text for a full description.

azimuthal flow are sufficiently small that we neglect them for the purposes of the torque balance analysis.

In equilibrium, we denote the axisymmetric interior azimuthal velocities, as observed in the laboratory frame, by $u_{1}(r)$ and $u_{2}(r)$. The subscript " 1 " refers to the upper layer, and " 2 " to the lower layer. The assumption of columnar (i.e. depth-independent) flow follows from the Taylor-Proudman theorem for sufficiently small Rossby number (Acheson, 1990). To within an additive constant, the lower layer depth field, $h(r)$, corresponding to this flow is given by

$$
h(r)=\frac{1}{g^{\prime}} \int \frac{\left(u_{2}^{2}-u_{1}^{2}\right)}{r} \mathrm{~d} r .
$$

This interface height field is associated with radial pressure gradient forces in both layers which are exactly those required by fluid parcels executing circular motion at the specified speeds. The flow is assumed to be hydrostatic. The reduced gravity is $g^{\prime}=2 g\left(\rho_{2}-\rho_{1}\right) /\left(\rho_{2}+\rho_{1}\right)$, and the assumption is made that $g^{\prime} \ll g$. The external centrifugal effect gives a further contribution to the interface height shape, which is 
neglected in this analysis since it is weaker than the internal centrifugal effect - responsible for the term on the right of (1) - by a factor of $g / g^{\prime} \sim 100$ (Table I).

We now further assume that the azimuthal flow is one of layer-wise solid-body rotation, so that $u_{i}=r \Omega_{i}$, where $\Omega_{i}=\Omega+\Delta \Omega_{i}$ is the absolute rotation rate in layer $i \in\{1,2\}$. The validity of this assumption depends upon the size of the mean interface slope, and is discussed in Section 5. We may now evaluate the integrals in (1), to give

$$
h(r)=H+\frac{\left(\Omega_{2}^{2}-\Omega_{1}^{2}\right)\left(r^{2}-(5 / 2) a^{2}\right)}{2 g^{\prime}} .
$$

Conservation of volume, expressed as

$$
\int_{\theta=0}^{2 \pi} \int_{r=a}^{b} h(r) r \mathrm{~d} r \mathrm{~d} \theta=\pi\left(b^{2}-a^{2}\right) H
$$

has been imposed to determine the integration constant. For later use, we make the following definitions of the interface/sidewall intersection heights: $H_{+} \equiv h(a)=2 \mathrm{H}-$ $h(b)$ and $H_{-} \equiv h(b)=2 H-h(a)$. Fluid at the axisymmetric interface, $z=h(r)$, is assumed to rotate with angular velocity $\Delta \Omega_{\mathrm{I}}$ in the turntable frame, as shown in Fig. 5.

In (2), the interior rotation rates in the turntable frame, $\Delta \Omega_{1}$ and $\Delta \Omega_{2}$, are as yet unknown. In much of Hart's work, including Hart (1972, 1973, 1985), as well as in other studies including Bradford et al. (1981), these interior rotation rates are derived from the two-layer quasi-geostrophic equations, neglecting the influence of Stewartson layers at the sidewall boundaries and assuming a flat, horizontal interface. For the case of exactly equal viscosities, this calculation yields the simple result $\Delta \Omega_{2}=(1 / 4) \Delta \Omega$ and $\Delta \Omega_{1}=(3 / 4) \Delta \Omega$, which can be substituted into (2) to obtain an explicit expression for $h(r)$.

The assumptions of quasi-geostrophy, non-interacting Stewartson layers and a horizontal interface mean that this method can only be considered as a first approximation. The present calibration scheme requires a more accurate analysis, as will be justified in Section 3.3, where we show that the layer rotation rates obtained from our advanced calculation differ significantly from the simple result quoted above. King (1979) developed an alternative approach based on a balance of torques in each layer. He argued that, in equilibrium, the fluid interiors do not experience an angular acceleration, and so the net external torque on the interiors due to the shears across the boundary layers must be zero. Stewartson layers and ageostrophy are both included, but King assumed a flat, horizontal interface to make the calculation analytically tractable.

In the present problem, we specifically require a non-horizontal interface, as we would like the calibration curve to span as wide a range of interface heights as possible. We therefore now extend the torque balance calculation to include non-horizontal interface effects.

\subsection{Derivation of torques}

For the torque balance calculation we model each fluid layer as an inviscid interior region, making up the vast majority of the volume of the layer, surrounded on all 
sides by thin viscous boundary layers which serve to change the fluid velocity from its interior value to its no-slip boundary value. In the two-layer annulus, the boundaries are the lid, base and fluid interface (at which the boundary layers are Ekman (1905) layers), and the inner and outer cylindrical sidewalls (at which the boundary layers are Stewartson (1957) layers).

We expect, when the imposed lid rotation $\Delta \Omega$ is positive, that

$$
0<\Delta \Omega_{2}<\Delta \Omega_{\mathrm{I}}<\Delta \Omega_{1}<\Delta \Omega
$$

where $\Delta \Omega_{\mathrm{I}}$ is the angular velocity of the interface (see Fig. 5). Qualitatively, the upper layer interior is being acted upon by a prograde (anti-clockwise) stress due to the Ekman layer at the lid, and by retrograde (clockwise) stresses due to the Ekman layer above the interface and both upper layer Stewartson layers. The boundary layer at the lid is tending to spin the layer up, and the remaining three boundary layers are tending to spin it down. In the lower layer, it is the interfacial boundary layer which gives a positive angular velocity tendency, and the remaining three which give a negative contribution.

To simplify the following analysis we assume equal layer viscosities, $v$, and densities, $\rho$, both of which approximations are very good in the present context (see Table I). Neither viscosity differs from the mean viscosity (the value used in the model) by more than $10 \%$. The model would be made much more complicated by including the different viscosities, and it is not clear that the extra complexity would be justified by any significant gain in accuracy. In the limit of small Rossby number (see Section 5 for a discussion) the Ekman and Stewartson layer widths are, respectively,

$$
\delta_{\mathrm{E}}=\left(\frac{v}{\Omega}\right)^{1 / 2} \text { and } \quad \delta_{\mathrm{S}}=\left(\frac{a^{2} v}{\Omega}\right)^{1 / 4}
$$

For a typical rotation rate of $\Omega=1 \mathrm{rad} \mathrm{s}^{-1}$, we have $\delta_{\mathrm{E}}=1 \mathrm{~mm}$ and $\delta_{\mathrm{S}}=8 \mathrm{~mm}$.

In equilibrium, the interface does not accelerate, and therefore must feel no net torque due to the Ekman layers above and below it. The vertical shear in horizontal velocity across the upper interfacial Ekman layer must therefore equal that across the lower one, giving $\Delta \Omega_{\mathrm{I}}=\left(\Delta \Omega_{1}+\Delta \Omega_{2}\right) / 2$.

The shear stress on the upper layer interior by the vertical boundary $r=b$ is $-\rho v b \Delta \Omega_{1} / \delta_{\mathrm{S}}$, where the minus sign indicates that this stress represents a drag. A mean has been taken over the thin Stewartson layer, of width $\delta_{S}$, across which a velocity change of $b \Delta \Omega_{1}$ is achieved. This Stewartson layer has area $2 \pi b H_{+}$and is a distance $b$ from the rotation axis, and so it exerts a torque on the fluid of

$$
T_{\text {layer }=1, r=b}^{\text {Stewartson }}=-\frac{2 \pi \rho \nu \Delta \Omega_{1} H_{+} b^{3}}{\delta_{\mathrm{S}}} .
$$

Similar expressions are obtained for the torques $T_{\text {layer }=1, r=a}^{\text {Stewartson }}, T_{\text {layer=2, } r=b}^{\text {Stewartson }}$ and $T_{\text {layer=2, } r=a}^{\text {Stewartson }}$ due to the remaining three Stewartson layers.

The shear stress on the upper layer interior by the horizontal boundary $z=2 \mathrm{H}$ at radius $r$ is $\rho v r\left(\Delta \Omega-\Delta \Omega_{1}\right) / \delta_{\mathrm{E}}$. In this case the stress is dependent upon radius. 
An area element is $r \mathrm{~d} r \mathrm{~d} \theta$ and the distance from the axis is $r$, and so this Ekman layer exerts a torque on the fluid of

$$
\begin{aligned}
T_{\text {layer=1,z=2H }}^{\text {Ekman }} & =\int_{\theta=0}^{2 \pi} \int_{r=a}^{b} \frac{\rho \nu\left(\Delta \Omega-\Delta \Omega_{1}\right) r^{3}}{\delta_{\mathrm{E}}} \mathrm{d} r \mathrm{~d} \theta \\
& =\frac{\pi \rho \nu\left(\Delta \Omega-\Delta \Omega_{1}\right)\left(b^{4}-a^{4}\right)}{2 \delta_{\mathrm{E}}} .
\end{aligned}
$$

A similar expression is obtained for the torque $T_{\text {layer=2, } z=0}^{\text {Ekman }}$ on the lower layer interior due to the Ekman layer at the base.

The shear stress on the upper layer interior by the interface $z=h(r)$ at radius $r$ is $-\rho v r\left(\Delta \Omega_{1}-\Delta \Omega_{\mathrm{I}}\right) / \delta_{\mathrm{E}}$. In this case the area element is $\sqrt{1+(\mathrm{d} h / \mathrm{d} r)^{2}} r \mathrm{~d} r \mathrm{~d} \theta$ and the distance from the axis is $r$, and so this Ekman layer exerts a torque on the fluid of

$$
\begin{aligned}
T_{\text {layer=1, }=h}^{\text {Ekman }} & =-\int_{\theta=0}^{2 \pi} \int_{r=a}^{b} \frac{\rho v\left(\Delta \Omega_{1}-\Delta \Omega_{\mathrm{I}}\right) r^{3}}{\delta_{\mathrm{E}}} \sqrt{1+\left(\frac{\mathrm{d} h}{\mathrm{~d} r}\right)^{2}} \mathrm{~d} r \mathrm{~d} \theta \\
& \approx-\frac{\pi \rho \nu\left(\Delta \Omega_{1}-\Delta \Omega_{\mathrm{I}}\right)\left(b^{4}-a^{4}\right)}{2 \delta_{\mathrm{E}}} \sqrt{1+\left(\left.\frac{\mathrm{d} h}{\mathrm{~d} r}\right|_{r=b}\right)^{2}} .
\end{aligned}
$$

An approximation has been employed (without which further analytical progress becomes impossible) to replace the surd in the integrand of (10) with its value at $r=b$. This is justified because the $r^{3}$ factor is a much more strongly-varying function of $r$ than is the surd, which is at most linear in $r$. The variation of the surd throughout the integration range is therefore small compared with the variation of the $r^{3}$ term, and so we can treat the former as a constant, evaluated at $r=b$ since the $r^{3}$ factor heavily weights the integral towards the larger values of $r$. A similar expression to (11) is obtained for the torque $T_{\text {layer=2, } z=h}^{\mathrm{Ekman}}$ on the lower layer interior due to the Ekman layer at the interface.

\subsection{Torque balance equations}

We now write down expressions for the net torque in each layer, and equate them to zero in equilibrium to give

$$
T_{\text {layer=1,r=a }}^{\text {Stewartson }}+T_{\text {layer=1, } r=b}^{\text {Stewartson }}+T_{\text {layer=1, } z=2 H}^{\text {Ekman }}+T_{\text {layer=1, } z=h}^{\text {Ekman }}=0
$$

and

$$
T_{\text {layer=2, } r=a}^{\text {Stewartson }}+T_{\text {layer=2, } r=b}^{\text {Stewartson }}+T_{\text {layer=2, } z=0}^{\text {Ekman }}+T_{\text {layer=2, } z=h}^{\text {Ekman }}=0 .
$$


Equations (12)-(13) are two coupled nonlinear equations in the two unknowns $\Delta \Omega_{1}$ and $\Delta \Omega_{2}$. Rearranging, we may write the equations in matrix form,

$$
\left[\begin{array}{cc}
1+\alpha+\beta_{+} & -\alpha \\
-\alpha & 1+\alpha+\beta_{-}
\end{array}\right]\left[\begin{array}{c}
\Delta \Omega_{1} \\
\Delta \Omega_{2}
\end{array}\right]=\left[\begin{array}{c}
\Delta \Omega \\
0
\end{array}\right],
$$

where

$$
\alpha=\frac{1}{2} \sqrt{1+\left(\left.\frac{\mathrm{d} h}{\mathrm{~d} r}\right|_{r=b}\right)^{2}}, \quad \beta_{ \pm}=\frac{4}{15} \frac{\delta_{\mathrm{E}}}{\delta_{\mathrm{S}}} \frac{8 H_{ \pm}+H_{\mp}}{a} .
$$

The matrix equation is nonlinear because $H_{+}, H_{-}$and $\mathrm{d} h /\left.\mathrm{d} r\right|_{r=b}$ each depend upon $\Delta \Omega_{1}$ and $\Delta \Omega_{2}$ through (2). If we now make the horizontal interface assumption, which is $H_{+}=H_{-}=H$ and $\mathrm{d} h /\left.\mathrm{d} r\right|_{r=b}=0$, the equations linearize and we recover the results of the simpler torque balance calculation of King (1979). Additionally neglecting the influence of the Stewartson layers, by letting $\delta_{\mathrm{S}} \rightarrow \infty$ so that the torques $T_{\text {layer }=1,2, r=a, b}^{\text {Stewartson }} \rightarrow 0$, reduces the matrix equation to

$$
\left[\begin{array}{cc}
3 / 2 & -1 / 2 \\
-1 / 2 & 3 / 2
\end{array}\right]\left[\begin{array}{c}
\Delta \Omega_{1} \\
\Delta \Omega_{2}
\end{array}\right]=\left[\begin{array}{c}
\Delta \Omega \\
0
\end{array}\right],
$$

for which the solution is

$$
\left[\begin{array}{l}
\Delta \Omega_{1} \\
\Delta \Omega_{2}
\end{array}\right]=\left[\begin{array}{l}
\frac{3}{4} \Delta \Omega \\
\frac{1}{4} \Delta \Omega
\end{array}\right]
$$

This, as expected, is the simple quasi-geostrophic result referred to in Section 3.1.

In the present context, we use an iterative approach to solve the full, nonlinear matrix (14). We start by choosing $\Delta \Omega_{1}=\Delta \Omega_{2}=0$ as a first guess. Then we evaluate $H_{+}, H_{-}$ and $\mathrm{d} h /\left.\mathrm{d} r\right|_{r=b}$ for this $\Delta \Omega_{1}$ and $\Delta \Omega_{2}$, using (2), before evaluating the four matrix elements for this $H_{+}, H_{-}$and $\mathrm{d} h /\left.\mathrm{d} r\right|_{r=b}$. Finally, we solve (14a) to obtain an improved estimate for $\Delta \Omega_{1}$ and $\Delta \Omega_{2}$. If the original and improved solutions are not equal to within the required precision, we return to the start for a further iteration, this time using the improved estimate as a first guess.

The iterations were found to converge in almost all cases. The exceptions occurred when the product of $\Omega$ and $\Delta \Omega$ was very large - well outside the magnitudes encountered in most laboratory experiments - when a feature with a period of two iterations persisted in the equilibrated iteration series. In these cases there is presumably no equilibrium solution to the torque balance equations.

Figure 6 shows the results of the iteration calculation for the case $\Omega=3 \mathrm{rad} \mathrm{s}^{-1}$ and $\Delta \Omega=1 \mathrm{rad} \mathrm{s}^{-1}$. The fourth decimal place of the solutions is stable after around the 20th iteration. The converged solution for the lower layer rotation speed is $\Delta \Omega_{2}=0.23 \mathrm{rad} \mathrm{s}^{-1}$, quite close to $0.25 \mathrm{rads}^{-1}$ which is the corresponding simple quasi-geostrophic result. However, the converged solution for the upper layer is $\Delta \Omega_{1}=0.42 \mathrm{rad} \mathrm{s}^{-1}$, much smaller than the quasi-geostrophic result of $0.75 \mathrm{rad} \mathrm{s}^{-1}$. The influence of ageostrophy, Stewartson layer drag and a sloping interface can 


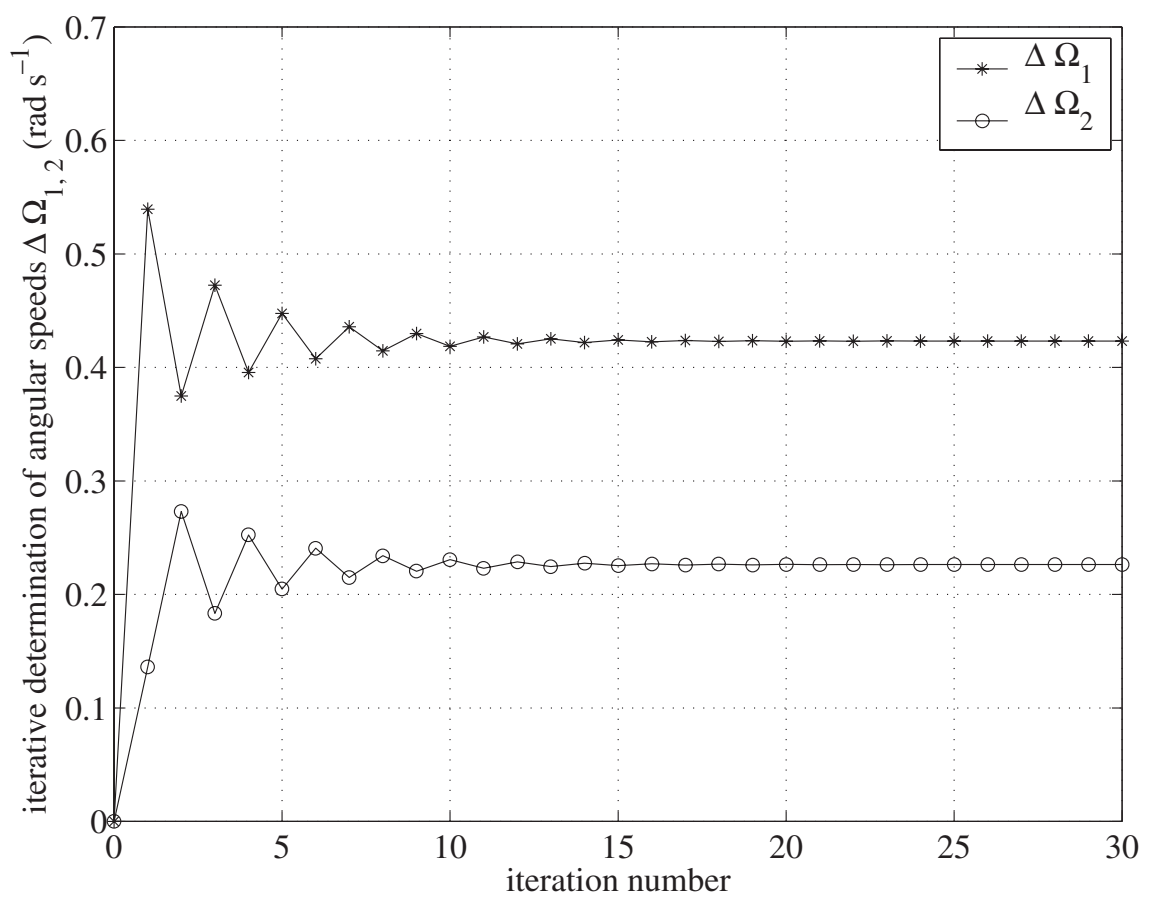

FIGURE 6 Results of the iterative numerical solution of the nonlinear matrix equation (14) for $\Delta \Omega_{1}$ and $\Delta \Omega_{2}$, for the case $\Omega=3 \mathrm{rad} \mathrm{s}^{-1}$ and $\Delta \Omega=1 \mathrm{rads}^{-1}$.

therefore significantly alter the equilibrium rotation rates, and it is important that their effects are included for an accurate calibration curve, as suggested at the start of this section.

\section{DERIVATION OF CALIBRATION CURVES}

We are now in a position to derive calibration curves. An image from a laboratory experiment, showing the equilibrated axisymmetric flow which is attained for the case $\Omega=0.77 \mathrm{rads}^{-1}$ and $\Omega=1.87 \mathrm{rads}^{-1}$, is shown in Fig. 7. For these parameters, equivalent to Rossby and Froude numbers of 0.2 and 7.4, respectively, there is neither a baroclinic nor a Kelvin-Helmholtz instability. There are 106 pixels across the annular gap, and there will therefore be 106 points on the calibration curve we obtain. For each of these pixels, we determine the digitised red $(R)$, green $(G)$ and blue $(B)$ colour components using standard image-processing software. Since the frame grabber gives a digitization of eight bits per colour channel, the components are given as integers between 0 and $2^{8}-1=255$. We then use these to calculate the hue as a function of radius, where hue is defined in terms of $R, G$ and $B$ by

$$
\text { hue }=\cos ^{-1}\left\{\frac{2 G-R-B}{\sqrt{2\left[(R-G)^{2}+(R-B)^{2}+(G-B)^{2}\right]}}\right\} \text {. }
$$




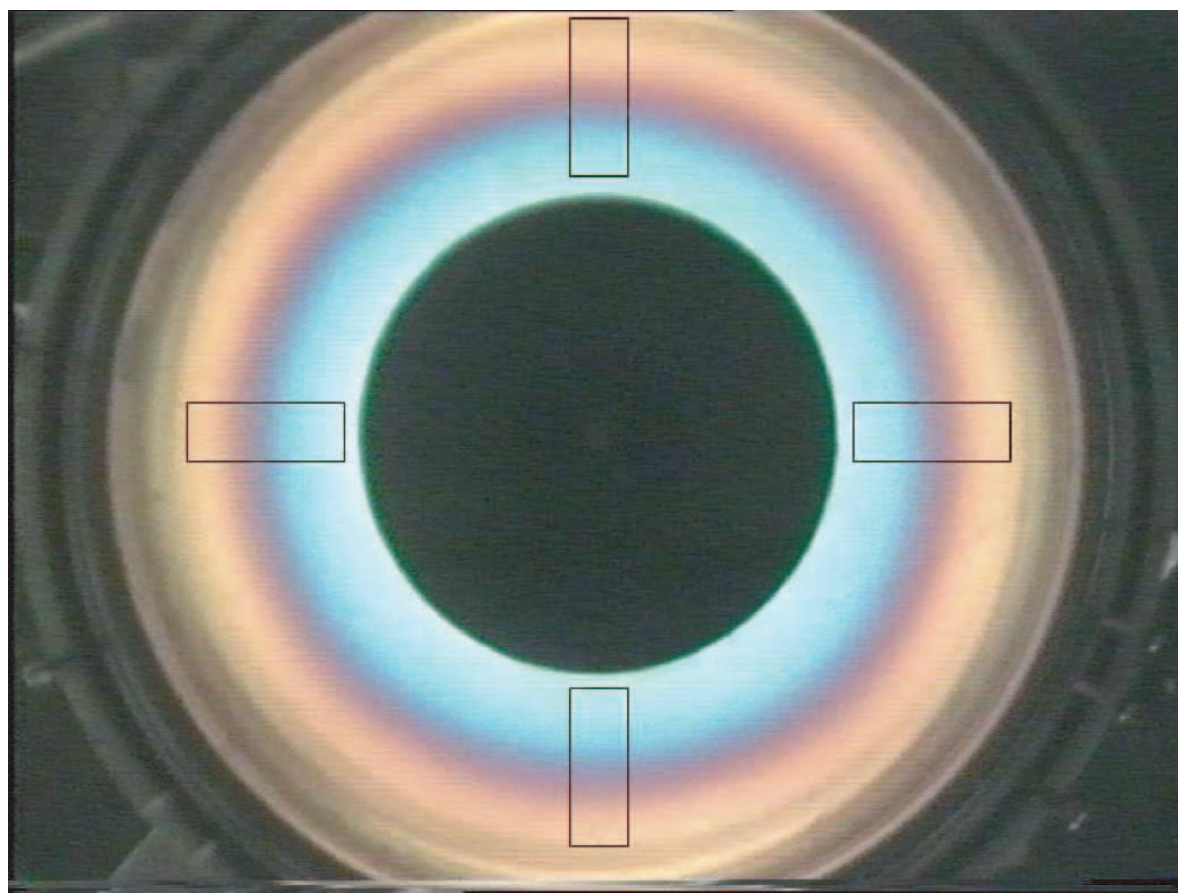

FIGURE 7 The first laboratory experiment image used for the calibration, showing the equilibrated flow in the case $\Delta \Omega=0.77 \mathrm{rad} \mathrm{s}^{-1}, \Omega=1.87 \mathrm{rad} \mathrm{s}^{-1}$. The four boxes, each measuring 106 pixels by 40 pixels, indicate the areas from which colour information is extracted.

Hue is an angle representing the dominant wavelength on a "colour wheel", for which $0^{\circ}$ corresponds to green, $120^{\circ}$ to blue and $240^{\circ}$ to red. Hue is one of the components of the hue, saturation, intensity (HSI) system for representing colours (Foley and Van Dam, 1982), which is an alternative to the red, green, blue ( $R G B$ ) system. Hue is an ideal calibration variable, as it is a monotonically-varying function of interface height over the range being considered, as we will shortly demonstrate. In the present context, this statement does not hold for the red, green, blue, saturation and intensity components. For example, it is clear from Fig. 7 that the intensity $(I=R+G+B)$ is large near the inner and outer sidewalls, with a minimum near mid-radius. A calibration curve based on this variable would therefore not be uniquely invertible. It is possible to restrict the range of radii under consideration, and then we may be able to use intensity as a proxy for interface height (Lovegrove et al., 2000), but it is clearly more attractive for the present purposes to use hue, for which the calibration is uniquely invertible across the entire radial span.

As indicated in Fig. 7, averages have been taken over the azimuthal angles corresponding to $0^{\circ}, 90^{\circ}, 180^{\circ}$ and $270^{\circ}$, in case the flow is not perfectly axisymmetric. Also as indicated in the figure, averages have been taken over 40 azimuthally-neighbouring pixels at each of these four angles, to reduce contamination of the signal by noise.

For the parameters corresponding to the calibration image in Fig. 7, the converged iterative solution to (14) is found to be $\Delta \Omega_{1}=0.34 \mathrm{rad} \mathrm{s}^{-1}$ and $\Delta \Omega_{2}=0.14 \mathrm{rad} \mathrm{s}^{-1}$. These rotation rates are substituted into (2) to obtain $h(r)$. Having both hue and 


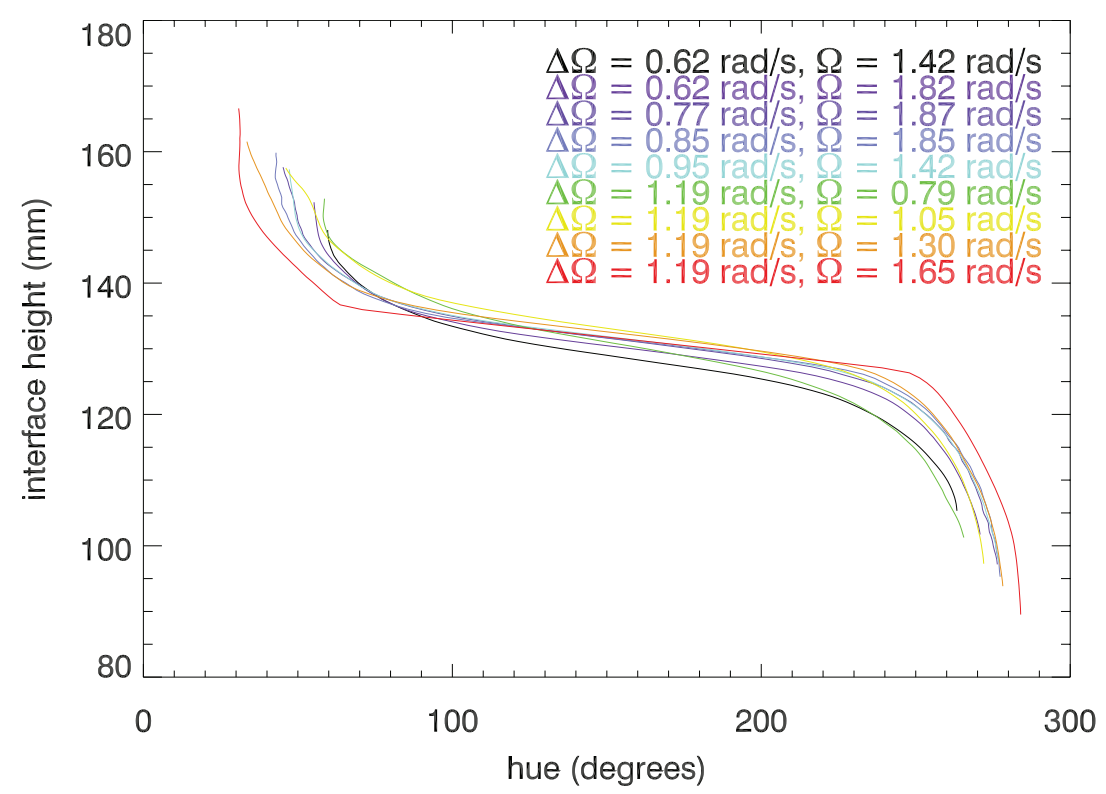

FIGURE 8 Family of calibration curves, each derived from different experimental conditions, as labelled. The curve derived from the image in Fig. 7 is included, together with eight others.

interface height as functions of radius allows us to eliminate the radius to obtain the relationship between hue and height. This procedure has been carried out using the calibration image of Fig. 7, plus eight further times using calibration images with different combinations of $\Omega$ and $\Delta \Omega$, corresponding to different axisymmetric flows. The family of calibration curves so obtained is shown in Fig. 8. There is good agreement between the curves, all of which have a characteristic S-shape. This validates the methods used to obtain the curves and shows that the calibration scheme is reliable. The curves span a large range of interface heights and hues, which have rarely been exceeded in the many laboratory experiments which we have performed using the present system.

As an example of an application of the calibration scheme, Fig. 9 shows the interface height field inferred from the image in Fig. 3, by projecting hue onto the average calibration curve of Fig. 8. Wave amplitudes, which previously had to be guessed by peering into the annulus lid, can now be measured. For example, we can easily determine that the amplitude at a radius of $r=100 \mathrm{~mm}$ is $13 \mathrm{~mm}$. An estimate of the precision of this measurement can be obtained by projection of the hue in Fig. 3 onto each of the nine calibration curves in Fig. 8 separately, from which we obtain nine amplitude measurements. The uncertainty, evaluated as the standard deviation, is found to be $1 \mathrm{~mm}$. Note that the uncertainty in inferred amplitudes is significantly less than the uncertainty in inferred interface heights, since the former is determined by the dispersion of the slopes of the nine curves, whereas the latter is determined by the dispersion of the values of the ordinate. It is clear that there is a better match between the slopes of the nine curves in Fig. 8, than there is between the ordinate values. The factors which limit the temporal, horizontal and vertical resolution of the retrieved interface maps are briefly discussed in Section 5. 


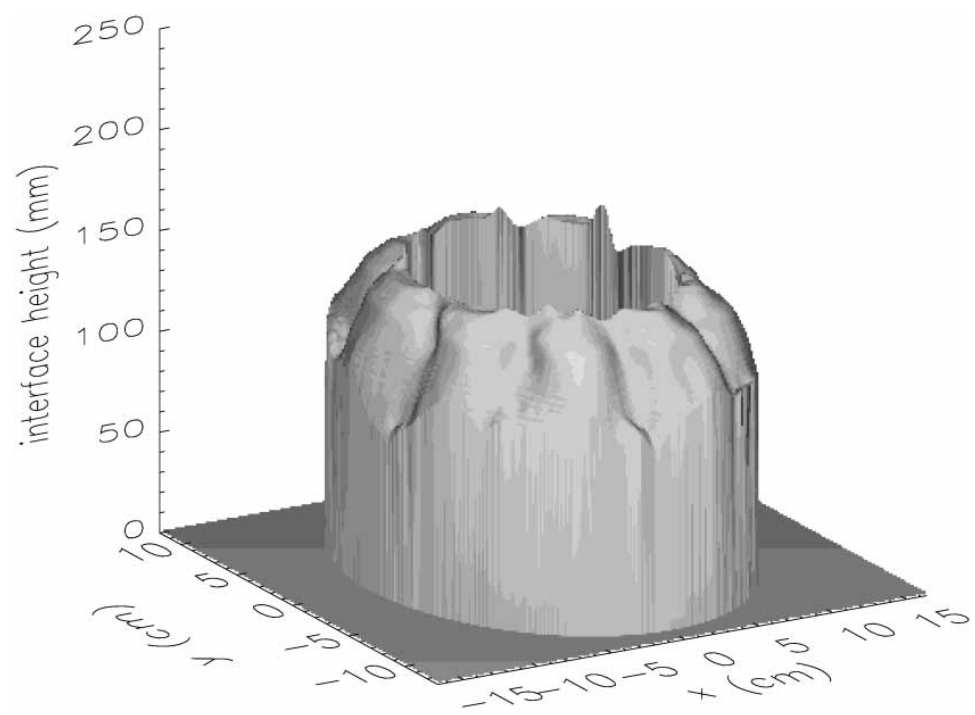

FIGURE 9 Reconstructed two-dimensional interface height, as inferred from the image in Fig. 3, by projecting the hue onto the mean of the calibration curves shown in Fig. 8.

\section{DISCUSSION}

We have described a technique for calibrating the flow visualization method originally proposed by Hart and Kittelman (1986). The method uses an optically-active fluid viewed between crossed polaroid sheets, to give a relation between colour and height. By writing down nonlinear torque balance equations for each of the two layers in the rotating annulus, we were able to iteratively determine the equilibrium interface height shape for the case of no dynamical instability. Combining this data with values of hue, digitized from the corresponding laboratory images using a frame grabber, has allowed us to derive a family of nine calibration curves as a consistency check. Finally, we have given an example of how the calibration scheme can be used to reconstruct the two-dimensional interface height for a flow exhibiting Kelvin-Helmholtz instability, and we have shown how wave amplitudes can easily be obtained from the map of retrieved heights.

In images such as that shown in Fig. 3, there are 106 pixels across the annulus gap of width $62.5 \mathrm{~mm}$, giving a horizontal resolution of $0.6 \mathrm{~mm}$. We have carried out further experiments in which a zoom lens is attached to the camera, giving 334 pixels across the annulus gap and a horizontal resolution of better than $0.2 \mathrm{~mm}$ (not shown). The camera frequency is $25 \mathrm{~Hz}$, giving a temporal resolution of $0.04 \mathrm{~s}$. The vertical resolution is limited by a phenomenon known as pixel jitter, which has contributions from the camera, video recorder and frame grabber, and which causes the digitised colour values to randomly vary about a mean state, even when the scene being filmed remains exactly the same. When inferring heights from a single image, pixel jitter limits the vertical resolution to around $1 \mathrm{~mm}$ (Williams, 2003). The effects of pixel jitter are weakened if averages are taken over neighbouring pixels in a single image, and/or over corresponding pixels in consecutive frames. This means that the vertical resolution can be significantly improved (by a factor of $\sqrt{N}$, for a mean taken over $N$ pixels) so long as a reduction in the horizontal and/or temporal resolution can be tolerated. 
We have recently used the calibration scheme to obtain the amplitudes of fast, inertia-gravity waves on scales of a few millimetres (Williams et al., 2003). An uncertainty would exist if the inertia-gravity wave amplitudes were measured using an in situ method, due to the possibility that the waves were being generated by an interaction between the measuring probe and the flow.

The Ekman and Stewartson boundary layer widths used for the torque balance analysis of Section 3 (given by (5) and (6)) are formally valid only for asymptotically small Rossby number. For small but finite Rossby number, nonlinear effects may cause modifications to the boundary layers, including instabilities (Bennetts and Hocking, 1973; Lingwood, 1997). However, Fig. 8 shows good agreement between calibration curves for Rossby numbers ranging from $\mathrm{O}(0.1)$ to $\mathrm{O}(1)$ (values outside this range cannot be used, because either a baroclinic instability is present or because the variation of interface height with radius is too small). Furthermore, the Ekman layer instability appears as vortex rolls in the form of spiral bands (Lilly, 1966) which are never observed in the present experiments, even with a Rossby number close to unity. These facts suggest that the asymptotic boundary layer theories remain valid slightly away from their formal domain of validity, with the implication that nonlinear boundary layer effects probably do not play a significant role in the calibration of this experiment.

In Section 3.1, we made an assumption of solid-body rotation in the fluid interiors. Strictly, this assumption is justified only for asymptotically small interface slopes. It is possible that the slopes reached in some of the present calibration experiments lie outside the formal domain of applicability of this assumption. However, the mean interface slopes in the nine calibration experiments (evaluated as $\left(H_{+}-H_{-}\right) /(b-a)$; see Fig. 5) vary by a factor of 2, with no apparent impact on the calibration curves of Fig. 8. Furthermore, we obtain good quantitative agreement between flows in the laboratory experiment and those in a quasi-geostrophic model (Williams, 2003), in which interface slopes are neglected and solid-body rotation is obtained. We therefore infer that our assumption of solid-body rotation is likely to be valid.

The calibration method described here in the context of a rotating annulus, could equally well be applied to the case of a two-layer liquid in a rotating cylinder, as originally used by Hart and Kittelman (1986). A slight modification would be needed in the torque balance analysis, by setting the inner sidewall radius to zero and removing the effects of the inner Stewartson layers. Similarly, the scheme could easily be applied to an annulus in which the outer sidewall radius was not equal to twice the inner sidewall radius, by relaxing the condition $b=2 a$. More generally, a torque balance analysis similar to that described herein could potentially be applied to the non-rotating case $(\Omega=0, \Delta \Omega \neq 0)$, though the boundary layers in this ageostrophic case would not be Ekman and Stewartson layers in their conventional forms, and so the boundary layer width formulae in (5) and (6) would need to be modified.

The calibration curves in Fig. 8 are quite general, depending only on the ratio of limonene to CFC-113 in the lower layer mixture and on the angle between the crossed polaroids. Now that the curves have been determined, in the present study, they can be used for the investigation of many other diverse fluid flow problems involving an interface. The method developed here would therefore seem to offer a convenient and non-invasive way of obtaining reliable, high-resolution interface height measurements in a wide variety of experimental fluid flow configurations. 


\section{Acknowledgments}

PDW acknowledges support under a research studentship from the UK Natural Environment Research Council, held at the University of Oxford, with award reference number GT04/1999/AS/0203. The authors wish to thank two anonymous referees for their helpful comments.

\section{References}

Acheson, D.J., Elementary Fluid Dynamics. Oxford University Press (1990).

Bennetts, D.A. and Hocking, L.M., "On nonlinear Ekman and Stewartson layers in a rotating fluid", Proc. R. Soc. Lond. A 333, 469-489 (1973).

Bradford, J., Berman, A.S. and Lundgren, T.S., "Nongeostrophic baroclinic instability in a two-layer rotating system", J. Atmos. Sci. 38, 1376-1389 (1981).

Ekman, V.W., "On the influence of the earth's rotation on ocean currents", Ark. Math. Astr. Fys. 2, $1-52$ (1905).

Fincham, A.M. and Spedding, G.R., "Low cost, high resolution DPIV for measurement of turbulent fluid flow", Exp. Fluids 23, 449-462 (1997).

Foley, J.D. and Van Dam, A., Fundamentals of Interactive Computer Graphics. Addison-Wesley Publishing Co., Massachusetts (1982).

Goldstein, R.J., Fluid Dynamics Measurements. Springer-Verlag (1983).

Greenspan, H.P., The Theory of Rotating Fluids. Cambridge University Press (1968).

Hart, J.E., "A Laboratory Study of Baroclinic Instability", Geophys. Fluid Dynam. 3, 181-209 (1972).

Hart, J.E., "On the behaviour of large-amplitude baroclinic waves", J. Atmos. Sci. 30, 1017-1034 (1973).

Hart, J.E., "A laboratory study of baroclinic chaos on the f-plane", Tellus 37A, 286-296 (1985).

Hart, J.E. and Kittelman, S., "A method for measuring interfacial wave fields in the laboratory", Geophys. Astrophys. Fluid Dynam. 36, 179-185 (1986).

King, J.C., Instabilities and Nonlinear Wave Interactions in a Two-layer rotating fluid, Ph.D. Thesis, University of Leeds (1979).

Lilly, D.K., "On the instability of Ekman boundary flow", J. Atmos. Sci. 23, 481-494 (1966).

Lingwood, R.J., "Absolute instability of the Ekman layer and related rotating flows", J. Fluid Mech. 331, 405-428 (1997).

Lovegrove, A.F., Read, P.L. and Richards, C.J., "Generation of inertia-gravity waves in a baroclinically unstable fluid", Q. J. R. Meteorol. Soc. 126, 3233-3254 (2000).

Proudman, I., "The almost-rigid rotation of viscous fluid between concentric spheres", J. Fluid Mech. 1, 505-516 (1956).

Read, P.L., “Applications of singular systems analysis to baroclinic chaos”, Physica D 58, 455-468 (1992).

Ruddick, B.R., "The colour polarigraph — a simple method for determining the two-dimensional distribution of sugar concentration", J. Fluid Mech. 109, 277-282 (1991).

Stewartson, K., "On almost rigid rotations", J. Fluid Mech. 3, 17-26 (1957).

Stewartson, K., "On almost rigid rotations: Part 2", J. Fluid Mech. 26, 131-144 (1966).

Takeda, Y., "Velocity profile measurement by ultrasonic doppler method", Exp. Therm. Fluid Sci. 10, 444-453 (1995).

Williams, P.D., Nonlinear Interactions of Fast and Slow Modes in Rotating, Stratified Fluid Flows. D. Phil. Thesis, Department of Physics, University of Oxford (2003).

Williams, P.D., Read, P.L. and Haine, T.W.N., "Spontaneous generation and impact of inertia-gravity waves in a stratified, two-layer shear flow", Geophys. Res. Lett. 30, 2255. doi:10.1029/2003GL018498 (2003) 